\title{
Integrating Detectors for Photoacoustic Imaging
}

\author{
Hubert Grün'1, Thomas Berer ${ }^{1}$, Karoline Felbermayer ${ }^{1}$, Peter Burgholzer ${ }^{1}$, \\ Markus Holotta ${ }^{2}$, Gerhard Zangerl ${ }^{3}$, Robert Nuster ${ }^{4}$ and Günther Paltauf ${ }^{4}$ \\ ${ }_{1}^{1}$ RECENDT Research Center for NonDestructive Testing GmbH, Linz \\ ${ }^{2}$ Department of Radiology, Innsbruck Medical University, Innsbruck \\ ${ }^{3}$ Computational Science Center, University of Vienna, Vienna \\ ${ }^{4}$ Department of Physics, Karl Franzens University, Graz \\ Austria
}

\section{Introduction}

The aim of medical imaging is an unerring diagnosis of diseases. Up to now several well established imaging modalities like e.g. computed tomography (CT), magnetic resonance tomography (MRT), single photon emission computed tomography (SPECT), positron emission tomography (PET) or ultrasound imaging (US) are known. Each imaging modality exhibits advantages and shortcomings. Computed tomography images the absorption of Xray quanta and is suitable for imaging bone structures, brain imaging, angiography (imaging of blood vessels) but involves ionising X-rays. The contrast mechanism in MRT is the relaxation time of excited protons and therefore this method images soft tissue and vessels (using a contrast agent with the drawback that it can trigger an allergic reaction of the human body) best. But MRT is an expensive technology; the huge magnetic field is not easy to shield and disqualifies some patients with old models of cardiac pacemakers and other metallic implants. A new imaging modality called magnetic particle imaging (MPI) which is just topic of research - uses also high magnetic fields for imaging. In this case the fields generated by magnetic nanoparticles are imaged. Nuclear techniques like SPECT or PET involve a radionuclide for imaging functional processes like the metabolic rate - which is for instance higher in cancerous tissue than in healthy organs. The radionuclide is attached to a specific molecule and distributed in the body during the blood flow. The radioactive decay measured by adequate detectors shows the spatial distribution of the incorporated radioisotope which is higher in cancerous tissue compared to healthy tissue. Although these are important imaging modalities for cancer screening the radioactive substances which are incorporated in the body are one drawback apart from the high costs per examination. Nuclear imaging techniques only image functional processes but no anatomical structures for which reason other complimentary techniques (e.g. CT) are necessary. Ultrasound imaging displays the backscattering of ultrasonic waves on a boundary layer between different tissues or organs. Although US is a cheap and safe imaging modality, its contrast mechanism is only related to changes in acoustic properties. Since cancer arises from neoplastic cells, the properties of the cancer and the surrounding tissue are almost identical in terms of acoustic contrast during the first stages of cancer 
development. So there is no distinct boundary and therefore imaging contrast is low. Only at a progressed, but often too late stage the tumor can be imaged by US. To conclude, these contrast mechanisms and imaging modalities show the requirement for an additional imaging technique, since cancer screening and other applications are not satisfactorily covered by the established techniques.

Breast cancer screening is an important topic as many surveys document. Up to now X-ray mammography is the gold standard in breast imaging although several false-positive (there seems to be a cancerous lesion while in fact there isn't any cancer) and false-negative (an existing cancer is not detected) findings happen ( $\mathrm{Xu} \&$ Wang, 2006). Investigation of new imaging modalities using alternative contrast mechanisms is motivated by the potential of earlier diagnosis and reduced invasiveness. Recently several optical methods have been further developed, e.g. diffuse optical imaging (DOI) with the advantage of using nonionizing light, but with the drawback of low spatial resolution due to strong optical scattering in tissue. Generally, all pure optical imaging techniques have limited spatial resolution at depths of a few millimetres (Boas, 2011). Since new medical imaging techniques for applications like early breast cancer detection need high spatial resolution, further ideas have to be developed. A new promising imaging modality that addresses the problems/needs mentioned above is photoacoustic imaging.

Photoacoustic imaging shows promise to overcome the challenge of imaging with high contrast - as known from pure optical imaging methods - and high spatial resolution in deeper tissue layers. Although the principle of photoacoustics was investigated in 1880 by Alexander Graham Bell (Bell, 1880), the technology to use optically generated acoustic waves for imaging became possible only with the introduction of short laser pulses with duration in the range of picoseconds to nanoseconds. Photoacoustic imaging uses short pulses of electromagnetic energy, such as short laser pulses, to illuminate the sample/tissue. The imaging modality is therefore different from that described in early publications about photoacoustics (Rosencwaig \& Busse, 1980; Wong et al., 1979), where thermal waves generated by modulated electromagnetic radiation at relatively low frequencies interact with features of a sample.

In photoacoustic tomography the excitation beam is expanded to illuminate the whole sample at once. Best imaging quality is achieved when the excitation is as homogeneous as possible, which means an illumination from all sides around the sample/tissue is best suited. Depending on the wavelength the electromagnetic wave penetrates the sample/tissue in the range from several micrometers to a few centimetres ( $\mathrm{Xu} \&$ Wang, 2006). Inside the sample/tissue the electromagnetic energy is absorbed according to the local electromagnetic absorption properties, causing local heating in the range of millikelvin. This quantity is called specific absorption rate (SAR) $[\mathrm{W} / \mathrm{kg}]$. Due to this absorption thermoelastic expansion occurs, which launches broadband ultrasonic waves - the photoacoustic signal. Thus photoacoustic imaging is based on the conversion of the absorbed light into ultrasound waves and permits to image optical contrast with ultrasonic resolution. Photoacoustic signals contain frequencies in the range from several kilohertz up to several megahertz depending on the size of the objects: the smaller the structures the higher the frequencies. Short laser pulses absorbed in a semitransparent sample/tissue generate an initial pressure distribution

$$
p_{0}(\mathbf{r})=\Gamma W(\mathbf{r})
$$


proportional to the volumetric density $W(\mathbf{r})$ of the locally absorbed electromagnetic energy and the Grüneisenparameter $\Gamma$. Efficient generation of photoacoustic signals is only possible if the acoustic confinement and the thermal confinement are fulfilled ( $\mathrm{Xu} \&$ Wang, 2006). The former requires that the heating time (i.e. the pulse duration) of a certain absorbing object should be shorter than the time an acoustic wave needs to escape from that object. Thermal confinement is easier met, because it requires the heating time to be shorter than the characteristic time of, the relatively slow, heat diffusion from the absorbing object. The excited photoacoustic signals that carry information about the optical absorption of the tissue sample can be detected outside the object either by conventional piezoelectric transducers (Xu \& Wang, 2006), novel detectors like optical point detectors (Zhang et al., 2009; Berer et al., 2010) or integrating detectors which are described in detail in section 2. Collecting signals from a sufficient number of different positions around the sample allows tomographic image reconstruction in two and three dimensions. In section 3 the mathematics of integrating detectors is explained and principle ideas of image reconstruction are presented.

Many biological and medical applications arise from the advantage of photoacoustic techniques to provide optical contrast measured at ultrasonic resolution. Because of the high optical contrast between haemoglobin in the blood and the surrounding tissue, it can be used to visualize single blood vessels as well as the blood distribution in whole organs. The formation of new blood vessels (angiogenesis) can be an indication for cancer formation hence imaging of angiogenesis is an important diagnostic method and could be done by photoacoustics. But photoacoustic imaging also shows promise for a number of other possible applications. For example brain imaging through an intact skull of a rhesus monkey was done by $\mathrm{Xu}$ et al. (Xu \& Wang IEEE, 2006). Another group showed functional and structural imaging of a rat's cortex using photoacoustic tomography (Wang et al., 2003). Especially in preclinical and clinical studies non-invasive in-vivo methods are of interest. Small animal imaging is integral to a variety of preclinical imaging applications with researchers monitoring changes in organs and tissues, for example in response to changed physiological or environmental influences. Researchers want to see how an organ is working inside the body. Ex-vivo analysis isolates the organs, while in-vivo imaging, of course, provides a much better picture of what is happening inside the body. Photoacoustic tomography (using a small animal imager) could therefore provide solutions for such research topics (Boas, 2011). In section 4 first biological and medical experiments done with different types of integrating line detectors are presented, including images of insects (ant, flea) and an ex-vivo mouse model with myocardial infarction.

\section{Integrating line detectors}

For medical applications fast imaging techniques are necessary - real time imaging is the ultimate goal. Therefore many detectors have to be arranged around the sample to collect data simultaneously. By using many finite size conventional piezo elements it is possible to obtain a closed detection surface around the sample with the drawback of missing space for a homogeneous illumination from all sides. One possible approach to overcome this drawback was presented by Buehler et al (Buehler et al. 2010) were a bundle of glass fibers between the detectors was used. The detector size influences the achievable spatial resolution: the smaller the detecting element the better is the resolution. Due to physical 
limitations it is not possible to miniaturize detectors arbitrary also the signal to noise ratio (SNR) gets worse for a smaller detector volume.

Because of these reasons new concepts for detectors were developed and investigated. Haltmeier et al. (Haltmeier et al., 2004) and Burgholzer et al. (Burgholzer et al, 2005) introduced integrating area and line detectors. Their size is at least $\sqrt{8} \cdot D$ larger than the imaged object, whereas $D$ is the diameter of a circle enclosing the sample and tangentialy touching the line detector. This way one can overcome the problem of miniaturizing the detector, which can be more easily done with optical approaches compared to piezo detectors. Moreover, integrating line detectors can be based on laser beams or optical fibers (as described in section 2.1) and therefore these detectors are no barrier for the illumination. The excitation laser beam can cross the detector without any disturbance.

An integrating detector integrates the pressure along two axes (area detector) or at least along one axis (line detector). An integrating area detector can be fabricated using a piezoelectrical film, e.g. made of PVDF (polyvinylidene fluoride), which offers better broadband sensitivity compared to the conventional piezo elements. Burgholzer et al. (Burgholzer et al., 2005) presented first measurements using a PVDF film integrating area detector. Unfortunately there are some drawbacks using PVDF. The high capacity in combination with the resistance of the electric circuit operates like a high pass filter and important frequencies get lost. In general illumination through PVDF films is also not possible although Nierderhauser et al. (Niederhauser et al., 2005) presented a transparent detector made of indium tin oxide (ITO). For an exact reconstruction one has to move the large area detector tangentially on the surface of a sphere enclosing the sample to collect data from all sides. Moving the detector this way is mechanically challenging. Dividing an integrating area detector into many integrating line detectors simplifies the movement of the detector and thus the setup. If many integrating line detectors are arranged in a circle around the sample only one rotation axis is needed as depicted in figure 1.

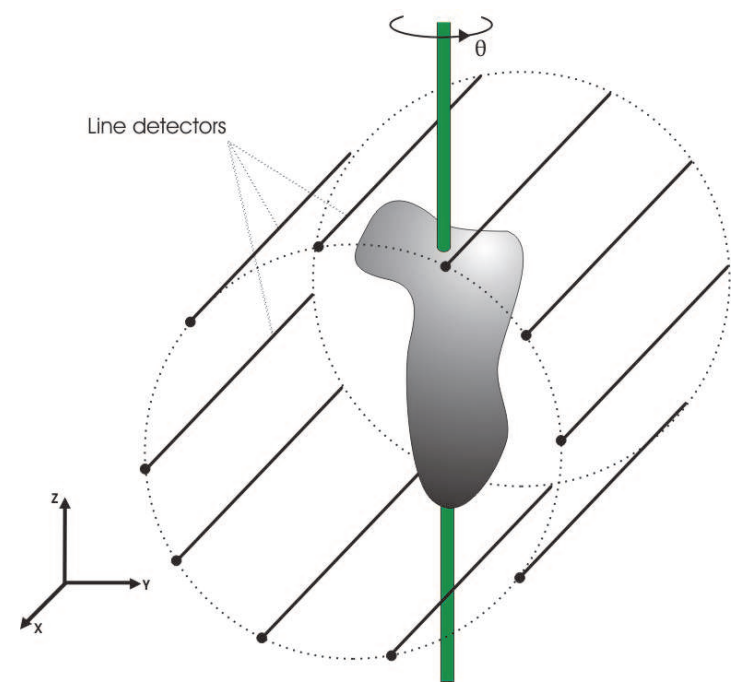

Fig. 1. Integrating line detectors arranged in a circle around the sample. In this mechanically simple setup only one rotation axis for three dimensional imaging is needed. 
Such an integrating line detector integrates the pressure along one dimension - i.e. along a line. Therefore it measures the integral of a cylinder with the radius $c \cdot t$ where $c$ is the speed of sound and $t$ is the time of flight. As described mathematically in section 3 this integrating behaviour results in a projection along the direction of the line detector. From a set of data acquired with line detectors arranged around the sample, e.g. in a circle (see figure 1) a projection image is obtained, which is analog to images taken with standard Xray mammography. For three dimensional imaging one has to rotate the sample (or the array of line detectors) to collect projection images of different angles. A subsequent inverse Radon transform reconstructs three dimensional images as explained in section 3. Some approaches of the implementation of optically integrating line detectors are presented in the following section.

\subsection{Implementation of integrating line detectors}

Since 2004 when Haltmeier et al. (Haltmeier et al., 2004) introduced the idea of integrating detectors several approaches of integrating line detectors have been investigated. A stripe of PVDF film was used as integrating line detector with some of the drawbacks mentioned before for piezoelectric sensors. As an alternative the use of optical interferometers as line detector has been proposed. In this approach a laser beam (or an optical fiber which guides a laser beam) being part of an interferometer is the sensitive element. The interferometer measures variations of the refractive index causing a variation of the change of the optical path length induced by the acoustic pressure (elasto-optic effect) which results in a change of the light intensity at the interferometer output. This section will give an overview about the implementation of different approaches of interferometric line detectors. The sensitive part of the interferometer - either the laser beam itself or the light guiding optical fiber - is immersed in a tank filled with water which operates as acoustic coupling medium for the wave propagation from the sample surface to the detector.

\subsubsection{Free-beam integrating line detectors}

\section{Free-beam Mach-Zehnder interferometer}

The simplest type of an optically integrating line detector is the Mach-Zehnder interferometer (MZI), which is a two-beam interferometer. Paltauf et al. implemented a freebeam MZI as integrating line detector and demonstrated first photoacoustic measurements (Paltauf et al., 2006). The schematic setup is depicted in figure 2. A continuous wave laser (633nm, HeNe-Laser) is split into two beams - one acting as reference beam and the other one as signal beam. Next to the signal beam the sample/tissue is located. Afterwards both beams are recombined and they interfere at the detecting photodiode with subsequent amplification. For stabilisation of the operating point (i.e. at quadrature condition) a piezoelectrically driven mirror in one path is used. Spatial resolution of an imaging setup is directly influenced by the size of the detector - in the case of a free-beam integrating line detector the diameter of the laser beam. A smaller diameter corresponds to better spatial resolution. By using lenses the laser beam is focused next to the sample/tissue to enhance spatial resolution. After the proof of principle (Paltauf et al., 2006) this kind of detector was further improved and the focus - and therefore the spatial resolution - was further enhanced. Paltauf et al. (Paltauf et al., 2009) characterized this integrating Mach-Zehnder line detector in detail. This characterization (shape of the signal from a point source, length 
of the line detector, directivity, and resolution) is also valid for all acoustically integrating line detectors.

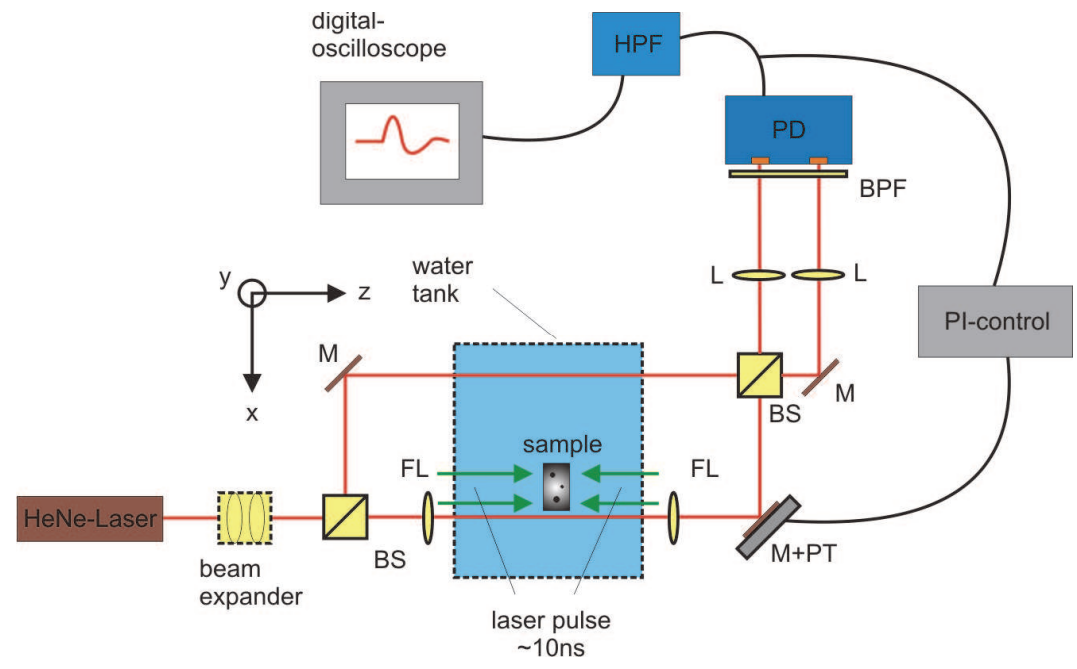

Fig. 2. Experimental setup for photoacoustic tomography using a Mach-Zehnder interferometer as integrating line detector. BS: beam splitter, M: mirror, L: lens, PD: photodetector, PT: piezo transducer, BPF: band pass filter, HPF: high pass filter, FL: focusing lens

\section{Free-beam Fabry-Perot interferometer}

Another approach for enhanced signal sensitivity is the use of a Fabry-Perot interferometer, which is a multi beam interferometer. A continuous laser beam is reflected several times between two semitransparent mirrors as depicted in figure 3 . The signal enhancing mechanism of this device can be understood in a way that the incoming pressure wave does not only act once on the laser beam but several times. Depending on the reflectivity of the mirrors the light circulation in the cavity is different which influences the sensitivity. Higher reflectivity results in a better sensitivity. Burgholzer et al. (Burgholzer et al., 2006) built a setup using a free-beam Fabry-Perot line detector as depicted in figure 3. A continuous

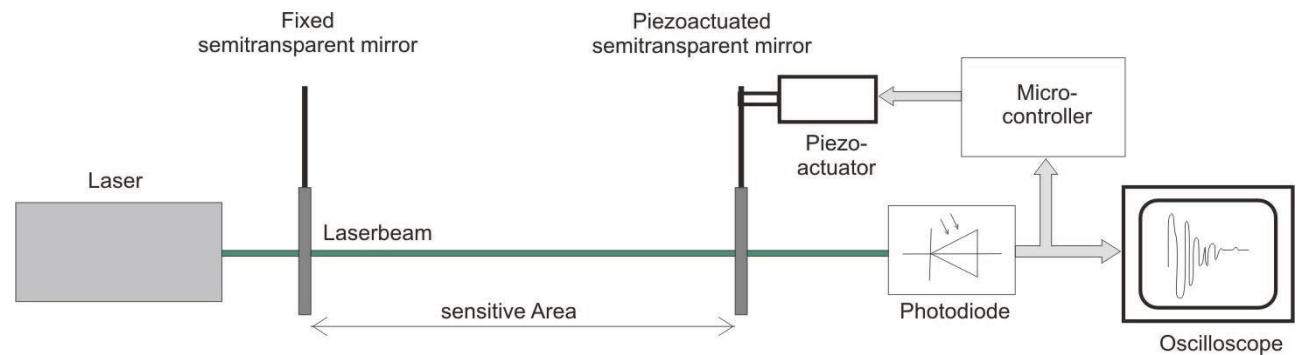

Fig. 3. Schematic of the used free-beam Fabry-Perot interferometer. A laser beam is reflected multiple times between two semitransparent mirrors and acts as integrating line detector. 
wave laser (532nm) was coupled into the Fabry-Perot cavity using two semitransparent mirrors with a reflectivity of approximately $95 \%$. The mirrors were realized by metalizing two microscope slides made of glass with thin layers of silver. On the opposite side of the Fabry-Perot cavity the modulated laser beam was detected by a photo diode with subsequent electrical amplification. For operation point stabilisation a piezo-electric actuator moved one of the semitransparent mirrors. In this case the laser beam was not focused and the diameter of the laser beam was constant along the line detector.

\subsubsection{Fiber-based integrating line detectors}

Both types of free-beam interferometers can also be realized in optical fibers. In this case the laser is coupled into and guided through an optical fiber. In general there are two types of optical fibers available that are applicable as sensor: glass optical fibers (GOF) and polymer optical fibers (POF). Glass optical fibers are standard components in telecommunication and are therefore cheap and reliable. Many components beside simple patch cords, e.g. beam splitters, attenuators, couplers and many more are available and easy to acquire. Recently also polymer optical fibers are used for short distance telecommunication. Due to high damping at the standard telecommunication wavelengths of $1310 \mathrm{~nm}$ and $1550 \mathrm{~nm}$ POFs are only used for the last few meters in the telecommunication chain (e.g. a network within a building). One advantage for standard applications is the low costs of POFs. For photoacoustic imaging polymer optical fibers are superior for realizing a detector compared to glass optical fibers as is described in detail in section 5 . Depending on how many modes (different possible paths of light) can propagate through an optical fiber one can distinguish between single mode fibers or multimode fibers. The smaller a core diameter of an optical fiber the fewer modes can propagate through the wave guide. As described above the fiber diameter also influences the achievable spatial resolution - thus single mode fibers (with the smallest outer diameter) are more convenient than multi mode fibers. Varying the types of interferometer (Mach-Zehnder interferometer or Fabry-Perot interferometer), the material of the optical fibers (glass or polymer) and the geometry of the core (single mode or multi mode fiber) result in various possible setups for fiber-based line detectors as described in the next section.

\section{Fiber-based Mach-Zehnder interferometer}

First, single mode glass optical fibers were used for a Mach-Zehnder setup. A laser with adjustable wavelength and a center wavelength of $1550 \mathrm{~nm}$ was coupled into a single mode glass fiber and split into two fibers. Analog to the free-beam Mach-Zehnder interferometer, one is the reference path and the other one is the signal fiber. The latter is placed in a tank filled with water next to the sample/tissue. At the sensitive region of the interferometer all outer layers of the fiber were removed, leaving only the core, cladding (both made of glass) and the inner surrounding polymer coating. The protection layers were removed to avoid damping of the pressure in the material. Both fibers - the reference path and the signal path - were combined afterwards and the interference of the laser beams was detected by a photodiode and a subsequent electrical amplifier (see figure 4). For operation point stabilization no mirrors had to be moved. Instead the wavelength of the laser was adjusted. Alternatively to the single mode GOF a perfluorinated graded index polymer optical fiber was used as signal path (Grün et al., 2009). The rest of the setup was identical with the single mode GOF setup described above. By using a more sensitive polymer fiber, signal detection could be improved. At the used wavelength of $1550 \mathrm{~nm}$ standard polymer optical fibers 
exhibit huge damping. Therefore a perfluorinated fiber - where the hydrogen molecules responsible for the damping are replaced by fluor molecules - was used. Unfortunately such perfluorinated fibers were only available as graded index fibers (a special case of multi mode fibers) with a core diameter of 50 microns.

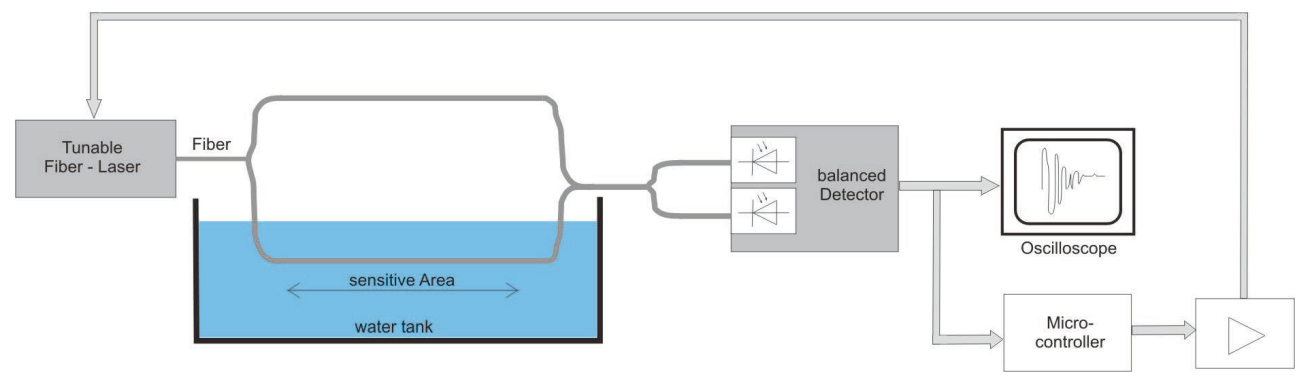

Fig. 4. Glass optical fibers (GOF) were used to realize a fiber-based Mach-Zehnder interferometer as optical line detector for photoacoustic imaging.

\section{Fiber-based Fabry-Perot interferometer}

As described earlier a Fabry-Perot interferometer is, because of the multiple reflections, more sensitive than a MZI. Therefore also fiber-based Fabry-Perot interferometers were realized. In glass optical fibers semitransparent mirrors are commonly realized by fiber Bragg gratings (FBG). A Bragg grating is a region in the fiber with a periodically changing refractive index (Dutton, 1989). Writing FBGs into GOFs is a standard procedure and these FBGs are commercial available. However, no stable Bragg gratings, which do not degrade in a seasonable amount of time, are available for polymer fibers. Alternatively the end faces of the fibers can be metalized by thin layers of metal (e.g. silver). This, however, comes at a cost of light absorption in the metallic layer. Fiber Bragg gratings are non-absorbing structures and therefore in general better than the metalized fiber end faces. A glass optical Fabry-Perot detector with Bragg gratings (Burgholzer et al., 2006) is depicted in figure 5. The laser beam is coupled into a single mode GOF. The detecting fiber has two FBGs with a reflectivity of approx. $99 \%$. The sensitive area, i.e. the area between the gratings, is uncoated - as described above for the fiber-based MZI detector. The photodiode and the amplifier are the same as used for the MZI setup. Operation point stabilization is again done by adjusting the wavelength of the laser. A similar setup was also realized using a perfluorinated graded index POF - the same used for the MZI (Grün et al, 2010 SPIE). In this case, because of lacking the possibility of stable Bragg gratings, the end-faces of the fiber were metalized.

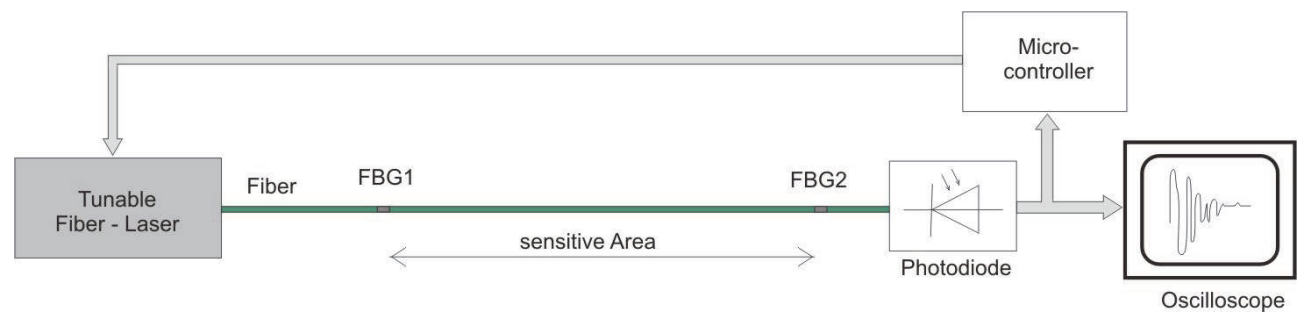

Fig. 5. Glass fiber-based Fabry-Perot line detector. Two fiber Bragg gratings (acting like semitransparent mirrors) form the cavity where the pressure transients are measured. 
After these implementations of optically integrating line detectors a brief introduction in the mathematics of integrating detectors is given in the next section.

\section{Mathematics of integrating detectors}

Photoacoustic imaging aims for reconstructing a three dimensional image of electromagnetic absorption properties from pressure data outside the object. The conversion of pressure measurements into a three dimensional image requires a model that describes acoustic wave propagation. Mathematically, the propagation of sound waves in tissue is modelled as a Cauchy Problem for the wave equation with constant sound speed. Denoting the thermoacoustic pressure by $p$ it satisfies the following Cauchy problem

$$
\begin{array}{cc}
\partial_{t}^{2} p(\mathbf{x}, t)=c^{2} \Delta p(\mathbf{x}, t) & (\mathbf{x}, t) \in R^{3} \times(0, \infty) \\
p(\mathbf{x}, 0)=f(\mathbf{x}) & \mathbf{x} \in R^{3} \\
\partial_{t} p(\mathbf{x}, 0)=0 \quad \mathbf{x} \in R^{3}
\end{array}
$$

for the three dimensional wave equation, where $\mathbf{x}$ denotes a point in a three dimensional space $R^{3}$ and $t \geq 0$ denotes the time variable. Further, we refer to $f(\mathbf{x}) \in C_{0}^{\infty}(\Omega)$ as the initial density or initial pressure of the considered Cauchy problem.

Commonly, in photoacoustic imaging it is assumed that acoustic pressure is known pointwise on a surface nearby the object and several reconstruction formulas have been derived that assume pointwise pressure data. However, in practice, acoustic pressure is measured by piezoelectric transducers, which can only provide approximate pointwise (pointlike) measurements. For this reason, the formulas above yield images with a spatial resolution that is essentially limited by the size of the piezoelectric transducers ( $\mathrm{Xu}$ et al., 2003). The size of a piezoelectric detector is typically a square with a side length of about $400 \mu \mathrm{m}$.

A first approach that tried to overcome the problem of finite aperture size of piezoelectric transducers has been given by M. Xu and L. Wang in (Xu et al., 2003). Therein the spatial blurring caused by detectors is modelled as a convolution with a point spread function of ultrasonic transducers and is used to improve reconstructions by deconvolution.

A practical approach, that tries to overcome the limitations due to finite aperture size of detectors, was proposed by Burgholzer et al. (Burgholzer et al., 2005), where linear and planar integrating, or shortly, linear and planar detectors were introduced. Planar, respectively, linear detectors measure a quantity that is proportional to the integral of acoustic pressure over a planar area or a line segment. Planar and linear detectors reduce the problems, caused by finite aperture size of piezoelectric transducers, because they approximate over a line or a plane very accurately. In the following we will describe the mathematical reconstruction process in the case of integrating measurement data.

\subsection{Planar detectors}

Planar detectors have been realized in experiments in (Burgholzer et al., 2005). Therein a planar piezoelectric film is used to approximate pressure measurements over a plane $E$. For 
simplicity we will assume for the moment that $E$ is the $y z$ plane. Then the measurements collected by the plane $E$ are given by

$$
G_{\text {planar }}(t)=\int_{E} p(0, y, z, t) d y d z
$$

where $p$ satisfies the wave equation. Haltmeier et al. (Haltmeier et al., 2004) derived a reconstruction algorithm for the measurement setup that is described and depicted in figure 6 .

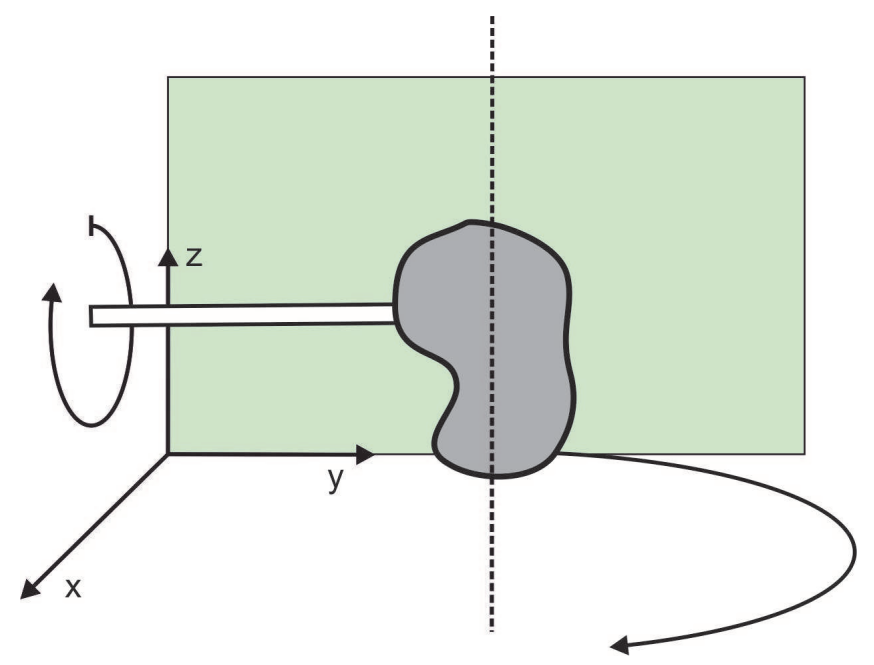

Fig. 6. The measurement procedure for planar detectors: After each measurement the object is rotated by a small angle increment and acoustic pressure is measured again. When the object has fully turned the planar detector is rotated around the dashed vertical axis and the whole measurement procedure is repeated. Once the planar detector has turned by 180 degree the process ends.

The reconstruction algorithm for planar detectors is based on a dimension reduction, that is based on the fact that the integrated pressure

$$
p_{\text {planar }}(x, t)=\int_{E} p(x, y, z) d y d z
$$

satisfies the one dimensional wave equation

$$
\begin{gathered}
\partial_{t}^{2} p_{\text {planar }}(x, t)-c^{2} \partial_{x}^{2} p_{\text {planar }}(x, t)=0 \\
p_{\text {planar }}(x, 0)=F(x)=\int_{E} f(x, y, z) d y d z \\
\partial_{t} p_{\text {planar }}(x, 0)=0
\end{gathered}
$$

By D'Alembert's formula the unique solution of the one dimensional wave equation is given by 


$$
p_{\text {planar }}(x, t)=\frac{1}{2}(F(x+c t)+F(x-c t))
$$

Thus, at the time $t$ the planar detector $E$ at $x=0$ measures

$$
G_{\text {planar }}(t)=p_{\text {planar }}(0, t)=\frac{1}{2} F(c t)
$$

where we have used that $f(-c t)$ vanishes since $f$ is only supported on one side of the detector. From the latter equation we see that a planar detector $E$ measures integrals of the original $f$ over planes parallel to $E$. Rotating the object at the detection planes as described in figure 6 yields the three dimensional Radon transform of the function $f$. Application of its inverse transform leads to a reconstruction of $f$.

\subsection{Linear detectors}

In the following we describe measurements that are collected by linear detectors as illustrated in figure 1. For convenience we will assume that the linear detectors are perpendicular to the $y z$ plane $E$. The measurements that are collected by the circular arranged array of lines in figure 1 are given by

$$
\mathrm{p}_{\text {linear }}(\mathrm{y}, \mathrm{z}, \mathrm{t})=\int_{\mathrm{L}} \mathrm{p}(\mathrm{x}, \mathrm{y}, \mathrm{z}, \mathrm{t}) \mathrm{dx}
$$

where $z, y$ lie on the circle $S_{R} \subset E$ (corresponds to the circle in figure 1). Equation (8) satisfies the two dimensional Cauchy problem.

$$
\begin{gathered}
\partial_{t}^{2} p_{\text {linear }}(\mathbf{x}, t)=c^{2} \Delta p_{\text {linear }}(y, z, t) \quad(y, z, t) \in R^{2} \times(0, \infty) \\
\mathrm{p}_{\text {linear }}(\mathrm{y}, \mathrm{z}, 0)=\mathrm{F}(\mathrm{y}, \mathrm{z})=\int_{\mathrm{L}} \mathrm{f}(\mathrm{x}, \mathrm{y}, \mathrm{z}) \mathrm{d} \mathrm{x} \quad \mathbf{x} \in \mathrm{R}^{3} \\
\partial_{t} p_{\text {linear }}(y, z, 0)=0 \quad \mathbf{x} \in R^{3}
\end{gathered}
$$

Unfortunately, the relation between $\mathbf{F}$ and the measurements $G_{\text {linear }}$ is more complicated as for the one dimensional wave equation. However, it is possible to obtain an exact relationship (Haltmeier et al., 2007) between $G_{\text {linear }}$ and $\mathbf{F}$ by means of integral transforms by

$$
\mathrm{H}_{\lambda}\left\{\mathrm{F}_{\lambda}\right\}(\omega)=\frac{(2 / \pi)^{1 / 2} \mathrm{C}\left\{\mathrm{G}_{\text {linear }, \lambda}\right\}(\omega)}{\omega \mathrm{J}_{|\lambda|}(\omega \mathrm{R})}
$$

whenever $\omega J_{|\lambda|}(\omega R) \neq 0$.

In this equation $H_{|\lambda|}$ denotes the Hankel transform of order $\lambda, C$ the cosine transform, $\omega$ the frequency variable of time and $F_{\lambda}, G_{\text {linear }, \lambda}$ are the Fourier coefficients of $F$ and $G_{\text {linear }}$ from their Fourier series expansion with respect to the angular variable. 
From the latter equation $F_{\lambda}$ can be computed by applying the inverse Hankel Transform. Accordingly $F$ can be computed from its Fourier series expansion if all the $F_{\lambda}$ are known. Note that $F$ consists of integrals over lines that are perpendicular to the plane $E$. Furthermore, the computations above would also hold for an arbitrary plane $E$. Thus one can obtain a family of projections $F_{\alpha}$ of the original function $f$ by rotating $E$ around the $z$ axis. For a fixed $z=z_{0}$ this family is nothing but the two dimensional Radon transform of the restriction of $f$ to the plane $z=z_{0}$. Applying the inverse Radon transform in each plane yields $f$.

\section{Biological and medical experiments with integrating line detectors}

Different types of line detectors and biological samples have been used to show the applicability of these detectors in the field of biomedical research. Some examples are given in the following section.

\section{Free-beam Mach-Zehnder interferometer for biological applications}

The free-beam Mach-Zehnder line detector was used, among others, for imaging of a flea. The small insect was chosen in order to test the imaging resolution and accuracy. To achievean adequate spatial resolution the sensing laser beam - which is the signal path of the interferometer - was focused to a diameter of the beam waist of $40 \mu \mathrm{m}$. For fixation the flea was embedded in gelatine which shows good impedance matching with the surrounding water of the setup. For one projection image data were acquired at 90 detector positions arranged on a semi circle with a radius of $5 \mathrm{~mm}$. 400 of such projection images
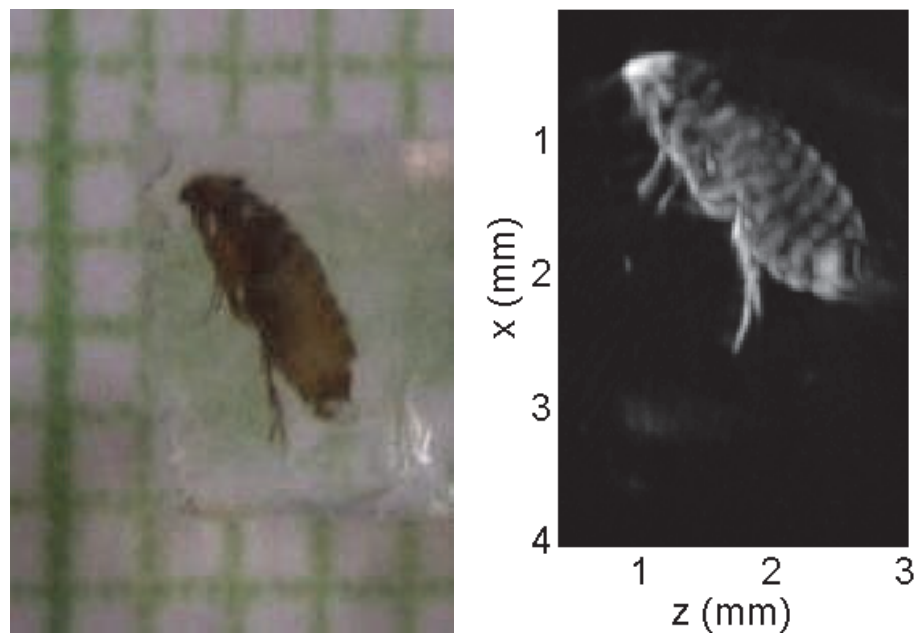

Fig. 7. Left: Photograph of a flea embedded in gelatine. Photograph is taken in front of a sheet of millimeter paper. The right picture shows a maximum amplitude projection (MAP) of the imaged flea in the xz-plane and was reconstructed using the universal back projection algorithm. 
distributed over an angle of $360^{\circ}$ were recorded. Laser pulses with a wavelength of $502 \mathrm{~nm}$ (Nd:YAG in combination with an OPO), and $8 \mathrm{~ns}$ pulse duration were used to excite photoacoustic signals in the flea. The radiant exposure was chosen with $10 \mathrm{~mJ} / \mathrm{cm}^{2}$ which is below the allowed maximum value for biological tissue $\left(20 \mathrm{~mJ} / \mathrm{cm}^{2}\right)$.

Looking at the reconstructed image (figure 7) of the flea one can clearly see the shape of the flea and the outer structures like the trotters. Also the striped structures at the back of the flea can be clearly identified.

\section{Free-beam Mach-Zehnder interferometer for preclinical applications}

Another study using the same type of setup was done a few months later. Nuster et al. (Nuster et al., 2010) and Holotta et al. (Holotta et al., 2011) presented first preclinical results on ex-vivo mouse hearts. They achieved a resolution in the range of 100 micrometers during imaging ex-vivo mouse hearts. Holotta et al. (Holotta et al., 2011) visualized ischemic areas within mouse hearts and compared it to already established imaging modalities like CT and MRT. In order to induce myocardial infarction, the left anterior descending artery has been ligated in-vivo in wild type mice. After varying survival periods the mice were sacrificed, the hearts were excised and immediately transferred into a $4 \%$ formaldehyde solution for conservation. Afterwards the hearts were embedded into 12\% Agar and provided with a small stick to enable mounting on a translation stage.

Photoacoustic signals were induced by 5 ns laser pulses $(10 \mathrm{~Hz}$ repetition frequency) at a wavelength of $750 \mathrm{~nm}$ where best results were obtained. The radiant exposure was maintained below the maximum value for biological applications $\left(20 \mathrm{~mJ} / \mathrm{cm}^{2}\right)$.

For data acquisition the sample was moved around the measurement beam in a $270^{\circ}$ arc and turned on its own axis continuously. A total of 283 projection images were recorded and used for three dimensional image reconstruction as shown in figure 8 .
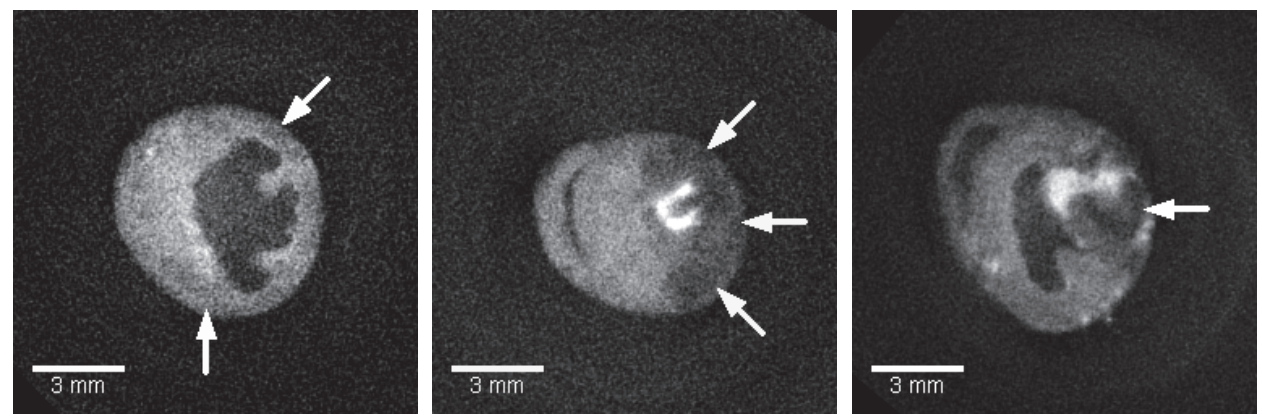

Fig. 8. Slices of mouse hearts - ischemic regions are marked by arrows. Left: mouse survived for 2 days. Middle: mouse survived for 3 days. Right: mouse survived for 4 days.

Due to the degradation of haemoglobin, the appearance of interstitial edema and various reparative processes (Friedrich, 2008; Kawasuji et al., 2000) the ischemic regions in the photoacoustic images (marked by arrows in figure 8) are characterized by lower absorption compared to the surrounding healthy muscle tissue in the myocardial wall. Agar - which is surrounding the ex vivo mouse heart - has a high water content and is, depending on its 
concentration, almost optically transparent. Therefore, it is hardly visible in the photoacoustic images shown in figure 8 .

\section{Fiber-based Fabry-Perot interferometer for biological applications}

Grün et al. (Grün et al., 2010) presented measurements of an ant utilizing a Fabry-Perot interferometer made of a single mode glass fiber as depicted in figure 5 . The reflectivity of the FBGs was $81 \%$ and the distance between the FBGs (i.e. the sensitive area) was $11,5 \mathrm{~cm}$. Data for one projection image were acquired at 200 line detector positions arranged in a circle (radius: $10 \mathrm{~mm}$ ) around the object. For three dimensional image reconstruction the sample was rotated and 25 projection images with an angle increment of 7.2 degrees were recorded. The ant was mounted on a nylon rod to allow rotation of the sample. In the lab where the tomography was carried out, unfortunately only an excitation wavelength of $532 \mathrm{~nm}$ was available. At this wavelength most of the absorption takes place in the outer layers of the ant, i.e. no signals from inside the ant were excited. Thus, only the surface of the ant was reconstructed. For illumination laser pulses from a frequency-doubled Nd:YAGLaser $(532 \mathrm{~nm})$ with a pulse duration of $6 \mathrm{~ns}$ and a repetition rate of $20 \mathrm{~Hz}$ were used. The pulse energy was adjusted to keep the radiant exposure below the allowed maximum value for biological tissue $\left(20 \mathrm{~mJ} / \mathrm{cm}^{2}\right)$.
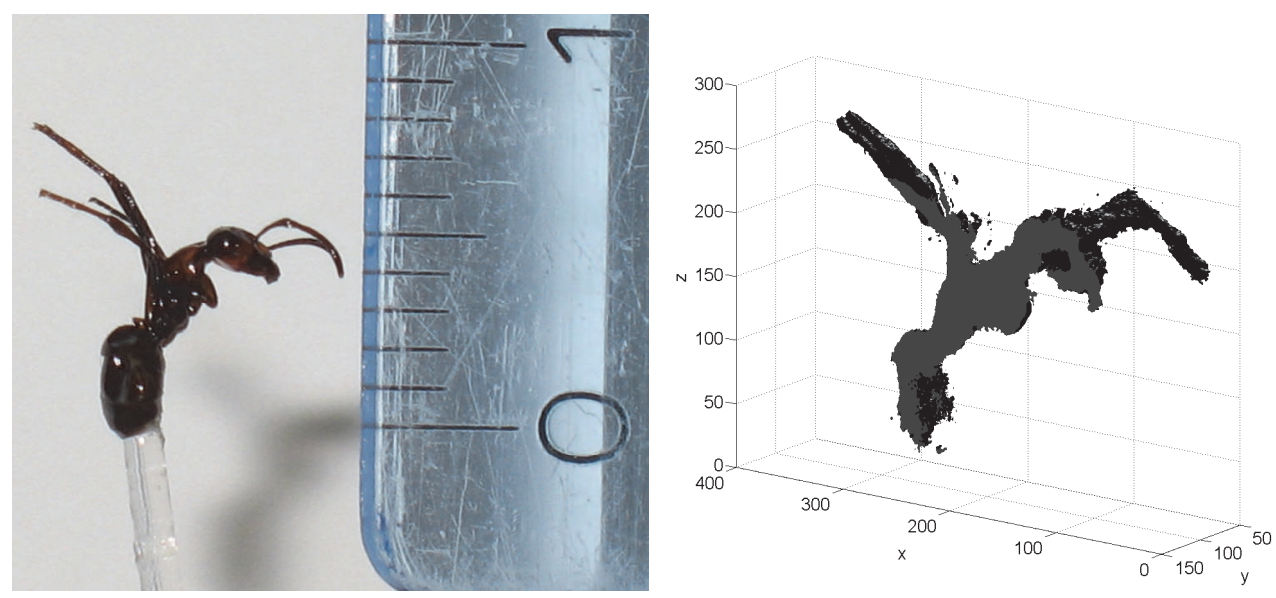

Fig. 9. On the left, a photograph of the ant - mounted on a nylon rod - can be seen. On the right a three dimensional reconstruction (using time reversal reconstruction) of the ant is displayed. 25 projection images, each consisting of 200 detector positions, were acquired for the whole tomographic image (Grün et al., 2010). 
In figure 9 one can see the reconstruction of the photoacoustically imaged ant. The reconstruction image is sliced in the middle of the ant. For image reconstruction a time reversal algorithm was used. One can distinguish the head (caput) with one feeler, the body (mesosoma) with two superposed trotters and the metasoma.

\section{Discussion}

In this section some advantages and disadvantages of the different approaches of optical line detectors are discussed as well as general considerations about optical line detectors.

Optical line detectors are more convenient compared with other detector types in general. These detectors are transparent - free-beam interferometers as well as fiber-based detectors - and therefore are no optical barrier for the excitation laser pulses. The sample can be illuminated across the transparent detectors, which would not be able using conventional piezo elements.

Using free-beam interferometers as well as fiber-based detectors one overcomes the directivity effects of piezo detector elements and finite size plane detectors. Due to the omni directional sensitivity of optical line detectors they can be easily scanned around an object without loss of sensitivity and signal quality.

Spatial resolution is an important issue in medical imaging. In photoacoustic imaging the bandwidth of the detector has influence on the spatial resolution. For smaller objects the emitted ultrasonic frequencies are higher. At the same time extended objects emit rather low frequencies. Thus it is necessary to collect the data from several kilohertz up to frequencies in the range of tens of megahertz to reconstruct all structures in an object. Interferometers as detector show a high bandwidth and are therefore appropriate for high resolution photoacoustic imaging.

Another parameter that influences the spatial resolution is the diameter of the detecting beam in an optical line detector. Smaller diameters correspond to a better spatial resolution. With a free-beam Mach-Zehnder interferometer one can achieve good spatial resolution by focusing the beam to a small diameter. But there are physical conditions which cannot be overcome. Focusing the beam to a tiny diameter leads to a short focal depth. Objects outside of this focal range can only be imaged with worse spatial resolution - the high resolution is only achievable for small objects. This issue was described by Paltauf et al. (Paltauf et al., 2009). Fiber-based line detectors, by contrast, provide constant diameter along the whole fiber - and therefore constant spatial resolution along the whole detector. Thus fiber-based line detectors are applicable for imaging of bigger objects.

Comparing the fiber Mach-Zehnder and the fiber Fabry-Perot interferometer the latter has several advantages in applications. Within a MZI all optical paths are sensitive. This means the signal path but also the reference path is sensitive to ultrasonic vibrations. Thus, disturbances from the environment and movements of the detector lead to additional noise. Only disturbances acting on both paths of the interferometer are compensated by the interferometer itself. By contrast, the FPI is only sensitive between the two mirrors, i.e. between the fiber Bragg gratings for the fiber-based approach. Therefore, the movement of the detector around the object and environmental influences are not that critical. Another advantage of the FPI is the higher sensitivity due to its multi beam 
interference. This leads to a steeper transfer curve, which is the relation between intensity change at the interferometer output and the optical phase variation caused by the acoustic pressure.

Operation point stabilization in free-beam interferometers can be easily done by a mirror mounted on a piezo actuator, which slightly changes the length of the light path. This is a simple and well known approach which was realized in the free-beam MZI. This operation point stabilization also was realized in the free-beam FPI but resulted in severe troubles. A piezo actuator outside the water tank was connected to one of the microscope slides (which was metalized and therefore acted like a semitransparent mirror). Due to the big surface of the microscope slide it showed resistance while moving in the water. In combination with the long distance (as can be seen in figure 3) between the end of the microscope slide (with the metalized part) and the piezo actuator this construction began to resonate and stable operation point stabilization was achievable only for low frequencies. Therefore, the MZI detector is more applicable for the free-beam approach.

For fiber-based interferometers the operation point stabilization can be done by two different approaches. One possibility is to tune the wavelength of the laser for operation point stabilisation. A tuneable laser - where the wavelength is changed in the range of a view of tens of picometers - was used for the interferometers presented in this work. This approach works well but is also an expensive method, beside of the fact that such suitable tuneable lasers are only available at the telecommunication standard wavelength of 1550 $\mathrm{nm}$. For some reasons shorter wavelengths for detection are be preferable. The damping in the used polymer fibers is much less for shorter wavelengths in the visible. A second reason is the higher phase shift of the detection light at the same pressure acting on the interferometer. This results in higher signal amplitudes and better SNR. Another approach for operation point stabilization, more equivalent to the free-beam operation point stabilization, is changing the optical path length. This can be done by electro-optical modulators which change the refractive index of the fiber or by piezo elements which change the length of the fiber. The latter can be realized by wrapping a fiber around an annular piezo element which changes the diameter or by stretching the fiber with a linear piezo stack changing its length. By using such an operating point stabilization one is independent of the detection wavelength. Furthermore, this is a cheap approach.

Another advantage of fiber-based detectors is that they offer the opportunity of arbitrarily shaped sensors. A line detector can be e.g. bent to an annular detector, as described for example by Berer et al. (Berer et al., 2009) and Grün et al. (Grün et al., 2011). This way new imaging opportunities arise. Only slight modifications of the detector are necessary as the detection concept is the same. Having biomedical applications in mind, optical line detectors have another advantage. Fiber-based detectors, especially those made of polymer, have excellent compatibility with organic material, thus giving them great potential for biomedical applications (Peters, 2011). This is important although the fiberbased detector is not in direct contact with the tissue when used as line detector for tomography. Free-beam line detectors of course, as they exhibit no material, are of course also compatible for organic materials. Therefore, they are also a good solution as detector for biomedical imaging.

Nuster et al. (Nuster et al, 2009) presented a comparison of the different types of optically integrating line detectors. At this stage the free-beam MZI was the most sensitive line 
detector, but the potential of more sensitive fiber-based line detectors could be clearly seen. In this study also the higher sensitivity of a FPI compared to a MZI was shown. For this reason the fiber-based detectors were further developed and many different approaches were implemented. In general, polymer optical fibers are more sensitive than glass optical fibers. This has two reasons. First, due to the lower Young's modulus of the polymer a pressure acting on a polymer fiber results in greater deformation compared to a glass fiber. As the strain-optic constants are similar the change in refractive index and thus the optical path length is greater and the same pressure transient generates a bigger signal in a polymer fiber than in a glass fiber. Second, the polymer is better impedance matched to the surrounding water. Thus most of the ultrasonic energy is transmitted into the fiber, while for glass optical fibers a large part is reflected. Using a single mode fiber, as described in section 2.1, results in the highest achievable spatial resolution when using fiber-based detectors. Summarizing these facts a single mode polymer fiber-based FPI with high finesse would be the most sensitive fiber-based line detector for photoacoustic imaging. Unfortunately at the time when the measurements, presented in section 4, were carried out only multimode polymer fibers were available. A first drawback of using this multimode fiber - in this case a graded index multimode fiber with a core diameter of 50 microns - was the worse spatial resolution compared with the single mode glass fiber-based detector. Another drawback was the multimode behaviour itself. When coupling light into the multimode fiber - independent of the interferometer type - always several modes are excited. The operation point, however, is stabilized on one of these excited modes. Whenever the fiber is moved - or whenever it relaxes between the points where it is attached to the detector holder - a different mode pattern is formed at the fibers end and the coupling into the successive single mode fiber changes. This results in unstable conditions for operation point stabilization. However, the proof of principle was shown and two dimensional image was demonstrated (Felbermayer et al., 2011). For time consuming three dimensional imaging, however, this method is up to now probably too unstable and cannot compete with the single mode glass fiber-based FPI. Another drawback of the used POF-FPI setup is related to the metalized fiber end faces instead of FBGs. Up to now it is not possible to write stable FBGs into polymer. For some reasons the FBGs written in POFs vanish or lose reflectivity after a period of days or weeks (Harbach, 2008). A FBG is a non absorbing structure in the fiber, the thin layers of silver, however, are absorbing structures and therefore reduce signal intensity. A polymer optical fiber-based Fabry-Perot line detector with fiber Bragg gratings acting as semitransparent mirrors seems be the goal for a future implementation.

The following points are not particularly specific to the topic of integrating detectors but are generally important for developing photoacoustic tomography devices. They have to be also discussed in relation to the detectors described in this chapter. As described earlier the smaller an object is the higher the emitted frequencies are. Therefore, frequencies ranging from several kilohertz up to some tens of $\mathrm{MHz}$ have to be detected. Unfortunately, in imaging of tissue in biological and medical application the effect of frequency depending attenuation is rather high. Higher frequencies are more damped than lower frequencies. This results in the loss of information about small structures in a certain depth. Thus, it is necessary to choose between a high bandwidth, high-frequency detector for microscopic imaging with high spatial resolution in small objects (or within a superficial region of few 
millimetres in a larger object) and a low frequency detector for tomographic imaging with lower spatial resolution in a bigger volume. Of course compensation of frequency depending attenuation improves the reconstructed image quality. But frequencies damped below the noise level of the detector cannot be retrieved (Bauer-Marschallinger et al., 2009; Burgholzer et al., 2009; Burgholzer et al., 2010).

For image reconstruction often constant speed of sound in the whole sample is assumed. Obviously this is just an approximation, as in biological samples the speed of sound is spatially varying. For example, between bones and surrounding tissue the difference in sound speed is considerable - but also in different types of tissue the sound velocity is slightly changing. Ignoring the spatial varying sound velocity can result in blurred images (Muratikov, 2004; Grün et al., 2008). However, compensation of the spatially varying sound velocity is not trivial, as one has to know the exact velocity distribution. This can be obtained by another imaging modality. Another approach is compensation by iterative image reconstruction algorithms.

For biological and medical imaging a short data acquisition time is sometimes crucial. Up to now only one optical line detector was moved around the sample - or in the case of the freebeam line detector the sample was moved around the laser beam. To speed up imaging parallel data acquisition is necessary. Instead of one line detector moving around the object many detectors could be arranged in an array. This way the data for one projection image can ultimately be obtained by one laser pulse. Only the detector array, or alternatively the object, has to be rotated to acquire many projection images for three dimensional image reconstruction. Parallelisation should be easier for fiber-based line detectors than for freebeam interferometers, although concepts for the latter one are available, such as the use of a CCD camera (Nuster et al., 2010).

\section{Conclusion and outlook}

Photoacoutic tomography is a new imaging method which is attractive for medicine and biology because it is capable to provide a three dimensional image of electromagnetic absorption properties of biological tissue - which is dependent of the used wavelength without ionizing radiation. These properties are of considerable interest for medical diagnostics as they are related to the molecular composition of tissue and reveal its pathological condition. Furthermore photoacoustic imaging is a cheap imaging modality compared to well known techniques like CT or MRT. For this rather new imaging modality new detectors - like the presented integrating detectors - are developed. In conclusion one can state that the optical approach of integrating line detectors works quite well. Not only the proof of principle but also the applicability for biological and (pre-) clinical imaging using different types of optical integrating line detectors has been shown. Several advantages like the frequency range, the spatial resolution, or the insensitivity against electromagnetic disturbances from the environment justifies this development and further enhancements of sensitivity and spatial resolution.

Future work will focus on parallelization of many line detectors - independent of the kind of optical line detector that will be further developed - to speed up this technology and collect all data for one projection image by only one single laser pulse. For fiber optical detection another enhancement would be the use of single mode polymer fibers which are 
much more sensitive than glass optical fibers but do not exhibit stability problems as the multi mode optical fibers. Therefore improved signal-to-noise ratio is expected which further speeds up imaging as less signal averaging is required. Because of their smaller dimensions single mode fibers will further increase the spatial resolution compared to the multimode polymer fibers in use now. Inscribing fiber Bragg gratings into polymer optical fibers for Fabry-Perot detectors would reduce losses, which appear when using metalized fiber end faces. Last but not least excitation pulse lasers with a higher repetition rate in the range of kilohertz also would shorten imaging time and therefore push photoacoustic imaging towards real-time clinical imaging.

\section{Acknowledgment}

This work has been supported by the Austrian Science Fund (FWF), Project Nos. S10502N20, S10503-N20, S10505-N20, S10506-N20, S10508-N20, L418-N20 and TRP102-N20; by the European Regional Development Fund (EFRE) in the framework of the EU-program Regio 13; and by the federal state of Upper Austria.

\section{References}

Bauer-Marschallinger, J.; Berer, T.; Grün, H.; Camacho-Gonzales, F.; Burgholzer, P. (2009). Experimental Determination of Frequency Dependent Acoustic Attenuation for Photoacoustic Imaging. Proceedings of SPIE 2009 European Conferences on Biomedical Optics: Novel Optical Instrumentation for Biomedical Applications IV, Munich, Germany, 2009

Bell, A.G. (1880). On the Production and Reproduction of Sound by Light: the Photophone. American Journal of Science, Vol. 20, pp. 305-324

Berer, T.; Grün, H.; Hofer, C.; Burgholzer, P. (2009). Photoacoustic Microscopy With Large Integrating Optical Annular Detectors, Proceedings of SPIE 2009 European Conferences on Biomedical Optics: Novel Optical Instrumentation for Biomedical Applications IV, Munich, Germany, 2009

Berer, T.; Hochreiner, A.; Zamiri, S.; Burgholzer, P. (2010). Remote photoacoustic imaging on solid material using a two-wave mixing interferometer. Optics Letters, Vol. 35, 4151

Boas, G. (2011). Small Animals, Big Achievements, BioPhotonics, pp. 18-20

Buehler, A.; Herzog, E.; Razansky, D.; Ntziachristos, V. (2010), Video rate optoacoustic tomography of mouse kidney perfusion. Optics Letters, Vol. 35, pp. 2475-2477

Burgholzer, P.; Hofer, C.; Paltauf, G.; Haltmeier, M.; Scherzer, O. (2005). Thermoacoustic Tomography With Integrating Area And Line Detectors. IEEE Transactions on Ultrasonics, Ferroelectrics, and Frequency Control, Vol. 52, pp. 1577-1583

Burgholzer, P.; Hofer, C.; Matt, G.J.; Paltauf, G.; Haltmeier, M.; Scherzer, O. (2006). Thermoacoustic tomography using fiber based Fabry-Perot interferometer as an integrating line detector, Proceedings of SPIE 2006 Biomedical Optics: Photons Plus Ultrasound: Imaging and Sensing 2006, San Jose, California, USA, January 21-26, 2006 
Burgholzer, P.; Comacho-Gonzales, F.; Sponseiler, D.; Mayer, G.; Hendorfer G. (2009). Information Changes and Time Reversal For Diffusion-Related Periodic Fields. Proceedings of SPIE 2009 Biomedical Optics: Photons Plus Ultrasound: Imaging and Sensing 2009, San Francisco, California, USA, 2009

Burgholer, P.; Roitner, H.; Bauer-Marschallinger, J.; Paltauf, G. (2010). Image Reconstruction in Photoacoustic Tomography Using Integrating Line Detectors Accounting for Frequency-Dependent Attenuation. Proceedings of SPIE 2010 Biomedical Optics: Photons Plus Ultrasound: Imaging and Sensing 2010, San Francisco, California, USA, 2010

Dutton, H.J.R. (1998). Understanding Optical Communications, IBM, ISBN 0130201413, USA

Felbermayer, K.; Grün, H.; Berer, T.; Burgholzer, P. (2011). Operation point stabilization of fiber-based line detectors for photoacoustic imaging. Proceedings of SPIE 2011 European Conferences on Biomedical Optics: Novel Optical Instrumentation for Biomedical Applications V, Munich, Germany, 2011

Friedrich, M.G. (2008), "Tissue Characterization of Acute Myocardial Infarction and Myocarditis by Cardiac Magnetic Resonance," Jacc-Cardiovascular Imaging. Vol. 1, pp. 652.

Grün, H.; Nuster, R.; Paltauf, P.; Haltmeier, M.; Burgholzer, P. (2008). Photoacoustic Tomography of Heterogeneous Media using a Model-Based Time Reversal Method. Proceedings of SPIE 2008 Biomedical Optics: Photons Plus Ultrasound: Imaging and Sensing 2008, San Jose, California, USA, 2008

Grün, H.; Berer, T.; Nuster, R.; Paltauf, G.; Burgholzer, P. (2009). Fiber-Based Detectors for Photoacoustic Imaging, Proceedings of SPIE 2009 European Conferences on Biomedical Optics: Novel Optical Instrumentation for Biomedical Applications IV, Munich, Germany, 2009

Grün, H.; Berer, T.; Burgholzer, P.; Nuster, R.; Paltauf, G. (2010). Three-dimensional photoacoustic imaging using fiber-based line detectors. Journal of Biomedical Optics, Vol. 15, No 2

Grün, H.; Berer, T.; Pühringer, K.; Nuster, R.; Paltauf, G.; Burgholzer, P. (2010). Polymer Fiber Detectors for Photoacoustic Imaging. Proceedings of SPIE 2010 Biomedical Optics: Photons Plus Ultrasound: Imaging and Sensing 2010, San Francisco, California, USA, 2010

Grün, H.; Altmisdört, H. ; Berer, T.; Paltauf, G.; Zangerl, G. ; Haltmeier, M.; Burgholzer, P. (2009). Photoacoustic tomography with integrating fiber-based annular detectors, Proceedings of SPIE 2011 Medical Imaging: Ultrasonic Imaging and Signal Processing, Orlando, Florida, USA, 2011

Haltmeier, M.; Scherzer, O.; Burgholzer, P.; Paltauf, G. (2004). Thermoacoustic Computed Tomography With Large Planar Receivers. Inverse Problems, Vol. 20, pp. 1663-1673

Haltmeier, M.; Scherzer, O.; Burgholzer, P.; Nuster, R.; Paltauf, G. (2007). Thermoacoustic Tomography and the Circular Radon Transform: Exact Inversion Formula. Mathematical Models and Methods in Applied Sciences, Vol. 17, pp. 635-655 
Harbach, N.G. (2008). Fiber Bragg Gratings in Polymer Optical Fibers. PHD-Thesis, Ecole Polytechnique Federale de Lausanne, Switzerland

Holotta, M.; Grossauer, H.; Kremser, C.; Torbica, P.; Volkl, J.; Degenhart, G.; Esterhammer, R.; Nuster, R.; Paltauf, G.; Jaschke, W. (2011), Photoacoustic tomography of ex vivo mouse hearts with myocardial infarction. Journal of Biomedical Optics Vol. 16, No 3

Kawasuji, M.; Ikeda, M.; Sakakibara, N.; Fujii, S.; Tomita, S.; Watanabe, Y. (2000), "Nearinfrared monitoring of myocardial oxygenation during ischemic preconditioning," Ann. Thorac. Surg. Vol. 69, pp. 1806.

Muratikov, K.L. (2004). Laer photoacoustic imaging of inhomogeneous objects. Technical Physics Letters, Vol. 30, pp. 956-958

Niederhauser J.J.; Jaeger, M.; Hejazi, M.; Keppner, H.; Frenz, M. (2005). Transparent ITO coated PVDF transducer for optoacoustic depth profiling. Optics Communications, Vol. 253, pp. 401-406

Nuster, R.; Gratt, S.; Passler, K.; Grün, H.; Berer, T.; Burgholzer, P.; Paltauf, G. (2009). Comparison of Optical and Piezoelectric Integrating Line Detectors, Proceedings of SPIE 2011 Biomedical Optics: Photons Plus Ultrasound: Imaging and Sensing 2009, San Francisco, California, USA, 2009

Nuster, R.; Holotta, M.; Kremser, C.; Grossauer, H.; Burgholzer, P.; Paltauf, G. (2010). Photoacoustic microtomography using optical interferometric. Journal of Biomedical Optics, Vol. 15, No 2

Nuster, R.; Zangerl, G.; Haltmeier, M.; Paltauf, G. (2010). Full Field Detectiron in Photoacoustic Tomography. Optics Express, Vol. 18, pp. 6288-6299

Paltauf, G.; Nuster, R.; Haltmeier, M.; Burgholzer, P. (2006). Photoacoustic tomography using a Mach-Zehnder interferometer as acoustic line detector. Applied Optics, Vol. 46, pp. 3352-3358

Paltauf, G.; Nuster, R.; Burgholzer, P. (2009). Characterization of integrating ultrasound detectors for photoacoustic tomography. Journal of Applied Physics, Vol. 105

Peters, K. (2011). Polymer optical fiber sensors - a review. Smart Materials and Structures, Vol.20

Rosencwaig, A; Busse, G (1980). High resolution photoacoustic thermal wave microscopy. Applied Physics Letters, Vol. 36, pp. 725-727

Wang, X; Pang, Y; Ku, G; Xie, X; Stoica, G; Wang, L. (2003). Noninvasive laser-induced photoacoustic tomography for structural and functional in vivo imaging of the brain. Nature Biology, Vol. 21 (7)

Wong, Y.H.; Thomas, R.L.; Pouch, J.J. (1979). Subsurface structures of solids by scanning photoacoustic microscopy. Applied Physics Letters, Vol. 35, pp. 368-369

Xu, M.; Wang, L. (2003). Analytic explanation of spatial resolution related to bandwidth and detector aperture size in thermoacoustic or photoacoustic reconstruction. Physical Review E, Vol. 67

Xu, M; Wang, L. (2006). Rhesus Monkey Brain Imaging through Intact Skull with Thermoacoustic Tomography. IEEE Transactions on Ultrasonics, Ferroelectrics, and Frequency Control, Vol. 53 (3) 
Xu, M; Wang, L. (2006). Photoacoustic imaging in biomedicine. Review of Scientific Instruments, Vol. 77

Zhang, E.Z.; Laufer, J.; Pedley, R.; Beard, P. (2009). In vivo high-resolution 3D photoacoustic imaging of superficial vascular anatomy. Physics in Medicine and Biology, Vol. 54, pp. 1035-1064 


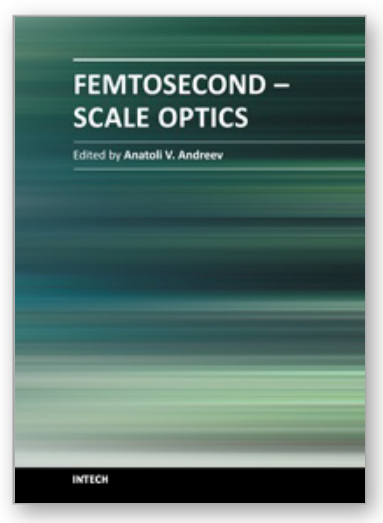

\author{
Femtosecond-Scale Optics \\ Edited by Prof. Anatoly Andreev
}

ISBN 978-953-307-769-7

Hard cover, 434 pages

Publisher InTech

Published online 14, November, 2011

Published in print edition November, 2011

With progress in ultrashort ultraintense laser technologies the peak power of a laser pulse increases year by year. These new instruments accessible to a large community of researchers revolutionized experiments in nonlinear optics because when laser pulse intensity exceeds or even approaches intra-atomic field strength the new physical picture of light-matter interaction appears. Laser radiation is efficiently transformed into fluxes of charged or neutral particles and the very wide band of electromagnetic emission (from $\mathrm{THz}$ up to $\mathrm{x}$-rays) is observed. The traditional phenomena of nonlinear optics as harmonic generation, self-focusing, ionization, etc, demonstrate the drastically different dependency on the laser pulse intensity in contrast the well known rules. This field of researches is in rapid progress now. The presented papers provide a description of recent developments and original results obtained by authors in some specific areas of this very wide scientific field. We hope that the Volume will be of interest for those specialized in the subject of laser-matter interactions.

\title{
How to reference
}

In order to correctly reference this scholarly work, feel free to copy and paste the following:

Hubert Grün, Thomas Berer, Karoline Felbermayer, Peter Burgholzer, Markus Holotta, Gerhard Zangerl, Robert Nuster and Günther Paltauf (2011). Integrating Detectors for Photoacoustic Imaging, FemtosecondScale Optics, Prof. Anatoly Andreev (Ed.), ISBN: 978-953-307-769-7, InTech, Available from: http://www.intechopen.com/books/femtosecond-scale-optics/integrating-detectors-for-photoacoustic-imaging

\section{INTECH}

open science | open minds

\section{InTech Europe}

University Campus STeP Ri

Slavka Krautzeka 83/A

51000 Rijeka, Croatia

Phone: +385 (51) 770447

Fax: +385 (51) 686166

www.intechopen.com

\section{InTech China}

Unit 405, Office Block, Hotel Equatorial Shanghai No.65, Yan An Road (West), Shanghai, 200040, China 中国上海市延安西路65号上海国际贵都大饭店办公楼405单元 Phone: +86-21-62489820

Fax: +86-21-62489821 
(C) 2011 The Author(s). Licensee IntechOpen. This is an open access article distributed under the terms of the Creative Commons Attribution 3.0 License, which permits unrestricted use, distribution, and reproduction in any medium, provided the original work is properly cited. 